\title{
Notes on fuzzy fractional Sumudu transform
}

\author{
Najeeb Alam Khan ${ }^{\mathrm{a}, *}$, Oyoon Abdul Razzaq ${ }^{\mathrm{b}}$, Muhammad Ayaz ${ }^{\mathrm{a}}$ \\ ${ }^{a}$ Department of Mathematics, University of Karachi, Karachi 75270, Pakistan. \\ ${ }^{b}$ Department of Humanities and Natural Sciences, Bahria University, Karachi 75260, Pakistan.
}

\begin{abstract}
In this paper, the analytical solutions of fuzzy fractional differential equations (FFDEs) are obtained by using the combination of fractional Sumudu transform (FST) and fuzzy calculus. In this regard, we extend the notation of FST to fuzzy fractional Sumudu transformation (FFST) and discuss its fundamental properties for the fuzzy-valued functions. Besides, a comprehensive study of FFST is also carried out for the different cases of Riemann-Liouville Hukuhara differentiability (H-differentiability) of fuzzy-valued functions. Moreover, to illustrate the capability and pertinence of this transform, solutions of some FFDEs are obtained, revealing its simplicity and efficiency.
\end{abstract}

Keywords: Fuzzy function, fractional differential equations, Sumudu transform, H-differentiability.

2010 MSC: 26A33, 34A08, 34A07.

(C)2018 All rights reserved.

\section{Introduction}

The amalgamation of fuzzy theory $[3,11,23,24]$ with fractional calculus $[2,8,17]$ has multiplied the practicality and expediency of calculus theory. Owing to the advantageous applications, both theories together have gained considerable attention in modeling different physical and engineering problems. Showing great concern, many authors have made abundant theoretical descriptions of fuzzy fractional calculus and developed numerous analytical and numerical methods for solution of FFDEs $[1,13,14,18$, 20].

Recently, connected to Fourier, bilateral, two sided and ordinary Laplace transforms, the Sumudu transform has begun to assert more fame for its distinct advantages and pragmatic applications. Subsequent to the introduction of Sumudu transform, initiated by Watugala [22], many other researchers documented valuable contributions to the theory and applications of Sumudu transform $[4,5,12,15,16,19]$. The main attribute of Sumudu transform lies in its unit preserving property and duality with Laplace transform that widens its application for solving various models of applied sciences. In addition, this transformation is constructed in context with fractional calculus to increase its capability to solve fractional differential models, as well. In this instance, several authors such as, Gupta et al. [10] proposed a

\footnotetext{
*Corresponding author

Email addresses: njbalam@yahoo.com (Najeeb Alam Khan), oyoon.abdulrazzaq@yahoo.com (Oyoon Abdul Razzaq), ayaz-maths@hotmail.com (Muhammad Ayaz)

doi: $10.22436 /$ jmcs.018.01.07
}

Received 2016-11-25 
new definition of fractional-order Sumudu transform for fractional differential functions, Bulut et al. [6] described the applications of Sumudu transform method to non-homogeneous fractional ordinary differential equations, Darzi et al. [7] solved fractional diffusion-wave equation by using Sumudu transform techniques, etc. $[9,21]$.

The aim of the present manuscript is to further widen the capability and applications of Sumudu transform on fuzzy fractional models. In this connection, we reformulate fractional Sumudu transform together with its properties using Riemann-Liouville fractional integral and H-differentiability of fuzzy functions to investigate a transform, named as fuzzy fractional Sumudu transform (FFST), for the solutions of FFDEs. Consequently, the proposed transform is applied to some examples of homogeneous and non-homogeneous FFDEs considered under Riemann-Liouville H-differentiability and thus analytical solutions are obtained.

\section{Preliminaries}

In this section, we define few preliminaries of fuzzy and fractional calculus theory which will be frequently exercised in the remaining paper.

\subsection{Fuzzy theory}

Let, $\mathfrak{R}$ be the set of real numbers and $\mathbf{I}=[0,1]$, then a set $\varphi$ with the membership function $\Psi_{\varphi}: \mathfrak{R} \rightarrow \mathbf{I}$, is said to be a fuzzy number if, it is normal, convex, upper semicontinuous, and compactly supported on $\mathfrak{R}$. Furthermore, $\varphi$ can also be represented by a closed interval of lower and upper functions $\varphi(\varepsilon)$ and $\bar{\varphi}(\varepsilon)$, respectively, i.e., $[\varphi(\varepsilon), \bar{\varphi}(\varepsilon)]$ for $0 \leqslant \varepsilon \leqslant 1$. Characteristically, $\varphi(\varepsilon)$ and $\bar{\varphi}(\varepsilon)$ are bounded monotonic increasing and monotonic decreasing functions, accordingly, both are left continuous on $(0,1]$ and also satisfy $\varphi(\varepsilon) \leqslant \bar{\varphi}(\varepsilon)$. For the remaining attempts, we will notate the space of all possible fuzzy numbers by $\boldsymbol{E}_{\mathrm{f}}$. The detailed descriptions of fuzzy set theory may also be found in [3, 11, 23, 24]. Moreover, let $\rho, \sigma \in \boldsymbol{E}_{\mathrm{f}}$, then the algebraic operations of fuzzy numbers can be defined as, for $0 \leqslant \varepsilon \leqslant 1$,

a. addition:

$$
[\rho \oplus \sigma]^{\varepsilon}=[\rho]^{\varepsilon} \oplus[\sigma]^{\varepsilon}=[\underline{\rho}(\varepsilon)+\underline{\sigma}(\varepsilon), \bar{\rho}(\varepsilon)+\bar{\sigma}(\varepsilon)] ;
$$

b. scalar multiplication: for $c \in \mathfrak{R}$,

$$
[c \rho]^{\varepsilon}=c[\rho]^{\varepsilon}= \begin{cases}{[c \underline{\rho}(\varepsilon), c \bar{\rho}(\varepsilon)],} & \text { if } c>0 \\ \{0\}, & \text { if } c=0 \\ {[c \bar{\rho}(\varepsilon), c \underline{\rho}(\varepsilon)],} & \text { if } c<0\end{cases}
$$

c. fuzzy multiplication:

$$
[\rho \odot \sigma]^{\varepsilon}=[\underline{r}(\varepsilon), \bar{r}(\varepsilon)],
$$

where

$$
\underline{r}(\varepsilon)=\min \{\underline{\rho}(\varepsilon) \underline{\sigma}(\varepsilon), \underline{\rho}(\varepsilon) \bar{\sigma}(\varepsilon), \bar{\rho}(\varepsilon) \underline{\sigma}(\varepsilon), \bar{\rho}(\varepsilon) \bar{\sigma}(\varepsilon)\}
$$

and

$$
\bar{r}(\varepsilon)=\max \{\underline{\rho}(\varepsilon) \underline{\sigma}(\varepsilon), \underline{\rho}(\varepsilon) \bar{\sigma}(\varepsilon), \bar{\rho}(\varepsilon) \underline{\sigma}(\varepsilon), \bar{\rho}(\varepsilon) \bar{\sigma}(\varepsilon)\} .
$$

\subsection{Fractional calculus}

Definition 2.1. The Riemann-Liouville integral of order $\beta>0$ for a fuzzy-valued function $\phi \in C_{f}[a, b] \cap$ $L_{f}[a, b]$, where $C_{f}[a, b]$ and $L_{f}[a, b]$ denote the space of all continuous fuzzy-valued functions and space of all Lebesgue integrable fuzzy-valued functions, respectively, is stated as,

$$
\mathcal{J}^{\beta} \phi(x)=\frac{1}{\Gamma(\beta)} \int_{0}^{x}(x-\zeta)^{\beta-1} \phi(\zeta) d \zeta, x>0 .
$$

Since, $[\phi(x ; \varepsilon)]=[\underline{\phi}(x ; \varepsilon), \bar{\phi}(x ; \varepsilon)]$, for $0 \leqslant \varepsilon \leqslant 1$, then we can also define fuzzy Riemann-Liouville 
integral of $\phi(x)$ in terms of its lower and upper functions for $x>0$ as,

$$
I^{\beta} \underline{\phi}(x ; \varepsilon)=\frac{1}{\Gamma(\beta)} \int_{0}^{x}(x-\zeta)^{\beta-1} \underline{\phi}(\zeta ; \varepsilon) d \zeta \quad \text { and } \quad I^{\beta} \bar{\phi}(x ; \varepsilon)=\frac{1}{\Gamma(\beta)} \int_{0}^{x}(x-\zeta)^{\beta-1} \bar{\phi}(\zeta ; \varepsilon) d \zeta,
$$

respectively.

Definition 2.2. A fuzzy-valued function $\phi \in C_{\mathrm{f}}[\mathrm{a}, \mathrm{b}] \cap \boldsymbol{L}_{\mathrm{f}}[\mathrm{a}, \mathrm{b}]$ is said to be Riemann-Liouville Hdifferentiable of order $0<\beta<1$ at $x_{0} \in(a, b)$, if there exists an element ${ }^{R L} \mathfrak{D}^{\beta} \phi\left(x_{0}\right) \in E_{f}$ such that for $\vartheta(x)=\frac{1}{\Gamma(1-\beta)} \int_{a}^{x} \frac{\phi(\zeta)}{(x-\zeta)^{\beta}} d \zeta$ and $h>0$ sufficiently small,



Let, fuzzy-valued function $\phi$ be I-differentiable if it is differentiable as in case (i) and II-differentiable if it is differentiable as in case (ii) and so on for the other cases.

Theorem 2.3. If $\phi \in C_{\mathrm{f}}[\mathrm{a}, \mathrm{b}] \cap \boldsymbol{L}_{\mathrm{f}}[\mathrm{a}, \mathrm{b}]$ is Riemann-Liouville H-differentiable of order $0<\beta<1$ at $\mathrm{x}_{0} \in(\mathrm{a}, \mathrm{b})$, then for $0 \leqslant \varepsilon \leqslant 1$,

i. ${ }^{\mathrm{RL}} \mathfrak{D}^{\beta} \phi\left(x_{0} ; \varepsilon\right)=\left[{ }^{\mathrm{RL}} D^{\beta} \phi\left(x_{0} ; \varepsilon\right),{ }^{\mathrm{RL}} D^{\beta} \bar{\phi}\left(x_{0} ; \varepsilon\right)\right]$, if $\phi$ is I-differentiable fuzzy-valued function;

ii. ${ }^{R L} \mathfrak{D}^{\beta} \phi\left(x_{0} ; \varepsilon\right)=\left[{ }^{R L} D^{\beta} \bar{\phi}\left(x_{0} ; \varepsilon\right),{ }^{R L} D^{\beta} \underline{\phi}\left(x_{0} ; \varepsilon\right)\right]$, if $\phi$ is II-differentiable fuzzy-valued function;

iii. ${ }^{R L} \mathfrak{D}^{\beta} \phi\left(x_{0}\right) \in \mathfrak{R}$, if $\phi$ is III-differentiable or IV-differentiable fuzzy-valued function,

where

$$
{ }^{\mathrm{RL}} D^{\beta} \underline{\phi}\left(x_{0} ; \varepsilon\right)=\left[\frac{1}{\Gamma(1-\beta)} \frac{\mathrm{d}}{\mathrm{d} x} \int_{\mathrm{a}}^{x} \frac{\phi(\zeta)}{(x-\zeta)^{\beta}} \mathrm{d} \zeta\right]_{x=x_{0}}
$$

and

$$
{ }^{\mathrm{RL}} D^{\beta} \underline{\phi}\left(x_{0} ; \varepsilon\right)=\left[\frac{1}{\Gamma(1-\beta)} \frac{\mathrm{d}}{\mathrm{d} x} \int_{\mathrm{a}}^{x} \frac{\phi(\zeta)}{(x-\zeta)^{\beta}} \mathrm{d} \zeta\right]_{x=x_{0}} .
$$

Equivalent theorems along with the detailed proofs of H-differentiability are found in $[3,18]$.

\subsection{Mittag-Leffler function}

The Mittag-Leffler function $\mathbb{E}_{\beta}(z)$ for $\beta>0$ and $z \in C$, where $C$ represents the complex numbers, is defined by the series representation,

$$
\mathbb{E}_{\beta}(z)=\sum_{\mathrm{k}=0}^{\infty} \frac{z^{\mathrm{k}}}{\Gamma(\mathrm{k} \beta+1)^{\prime}}
$$

whereas the generalized Mittag-Leffler function is expressed as,

$$
\mathbb{E}_{\beta, \mu}(z)=\sum_{k=0}^{\infty} \frac{z^{k}}{\Gamma(k \beta+\mu)} \quad \text { and } \quad \mathbb{E}_{\beta, \mu}^{\delta}(z)=\sum_{k=0}^{\infty} \frac{(\delta)_{k} z^{k}}{\Gamma(k \beta+\mu)},
$$

where, $(\delta)_{0}=1$ and for $\mathrm{k} \in \mathbf{N},(\delta)_{\mathrm{k}}=\delta(\delta+1) \cdots(\delta+\mathrm{k}-1)$. 


\section{Fuzzy fractional Sumudu transform}

The basic description of Sumudu transform, fractional Sumudu transform, and fuzzy Laplace transform together with their properties are thoroughly defined in $[4,12,20,22]$. Here, definitions of fractional Sumudu transform and fractional Laplace transform (FLT) under Riemann-Liouville integral with fuzzy functions are described. Furthermore, some properties such as duality and differentiation theorems are also investigated in fuzzy sense.

Definition 3.1. Let the fuzzy-valued function $\phi(x)$ be Riemann-Liouville integrable of order $\beta>0$ that disappears for negative values of $x$. Then the fuzzy fractional Sumudu transform of order $\beta>0$ for fuzzy-valued function $[\phi(x ; \varepsilon)]=[\phi(x ; \varepsilon), \bar{\phi}(x ; \varepsilon)]$ is defined as

$$
\mathrm{S}[\phi(x ; \varepsilon)]=[S[\phi(x ; \varepsilon)], S[\bar{\phi}(\mathrm{x} ; \varepsilon)]]
$$

for $0 \leqslant \varepsilon \leqslant 1$, where

$$
S[\underline{\phi}(x ; \varepsilon)]=\lim _{x \rightarrow \infty} \frac{1}{\Gamma(\beta)} \int_{0}^{x}(x-\zeta)^{\beta-1} \mathbb{E}_{\beta}\left(-\zeta^{\beta}\right) \underline{\phi}(p \zeta ; \varepsilon) d \zeta
$$

and

$$
S[\bar{\phi}(x ; \varepsilon)]=\lim _{x \rightarrow \infty} \frac{1}{\Gamma(\beta)} \int_{0}^{x}(x-\zeta)^{\beta-1} \mathbb{E}_{\beta}\left(-\zeta^{\beta}\right) \bar{\phi}(p \zeta ; \varepsilon) d \zeta .
$$

The symbol $\varepsilon_{\beta}$ is the Mittag-Leffler function described in Section 2.3 and S and $S$ are the FFST and FST operators for fuzzy-valued functions and real-valued functions, respectively.

Definition 3.2. Let $\phi(x)$ be the fuzzy-valued function and a Riemann-Liouville integrable of order $\beta>0$, which disappears for negative values of $x$. Then fuzzy fractional Laplace transform (FFLT) of order $\beta>0$ for fuzzy-valued function $[\phi(x ; \varepsilon)]=[\phi(x ; \varepsilon), \bar{\phi}(x ; \varepsilon)]$ is defined as

$$
\mathbb{L}[\phi(x ; \varepsilon)]=[L[\underline{\phi}(x ; \varepsilon)], L[\bar{\phi}(x ; \varepsilon)]]
$$

for $0 \leqslant \varepsilon \leqslant 1$, where

$$
L[\underline{\phi}(x ; \varepsilon)]=\lim _{x \rightarrow \infty} \frac{1}{\Gamma(\beta)} \int_{0}^{x}(x-\zeta)^{\beta-1} \mathbb{E}_{\beta}\left(-(p \zeta)^{\beta}\right) \underline{\phi}(\zeta ; \varepsilon) d \zeta
$$

and

$$
L[\bar{\phi}(x ; \varepsilon)]=\lim _{x \rightarrow \infty} \frac{1}{\Gamma(\beta)} \int_{0}^{x}(x-\zeta)^{\beta-1} \mathbb{E}_{\beta}\left(-(p \zeta)^{\beta}\right) \bar{\phi}(\zeta ; \varepsilon) d \zeta
$$

on the condition that integral exists, where $\mathbb{L}$ and $L$ are the FFLT and FLT operators for fuzzy-valued functions and real-valued functions, respectively.

Theorem 3.3. Let FFLT of a fuzzy-valued function $\phi(x)$ be $\mathbb{L}[\phi(x)]$ and $\mathbb{S}[\phi(x)]$ be its FFST, then

$$
\mathrm{S}[\phi(x)]=\frac{1}{p^{\beta}} \mathbb{L}[\phi(x)]=\frac{1}{p}
$$

and in case of its lower and upper functions,

$$
S[\underline{\phi}(x ; \varepsilon)]=\frac{1}{p^{\beta}} L[\underline{\phi}(x ; \varepsilon)]=\frac{1}{p} \quad \text { and } \quad S[\bar{\phi}(x ; \varepsilon)]=\frac{1}{p^{\beta}} L[\bar{\phi}(x ; \varepsilon)]=\frac{1}{p}{ }^{\prime}
$$

respectively. 
Proof. Following the description of FFST, and changing the variables of (3.1) and (3.2), i.e., $p \zeta=\zeta^{\prime}$ and $\mathrm{d} \zeta=\frac{\mathrm{d} \zeta^{\prime}}{\mathrm{p}}$, we obtain

$$
S[\underline{\phi}(x ; \varepsilon)]=\frac{1}{p^{\beta}} \lim _{x \rightarrow \infty} \frac{1}{\Gamma(\beta)} \int_{0}^{x}\left(x p-\zeta^{\prime}\right)^{\beta-1} \mathbb{E}_{\beta}\left(-\left(\frac{\zeta^{\prime}}{p}\right)^{\beta}\right) \underline{\phi}\left(\zeta^{\prime} ; \varepsilon\right) d \zeta
$$

and

$$
S[\bar{\phi}(x ; \varepsilon)]=\frac{1}{p^{\beta}} \lim _{x \rightarrow \infty} \frac{1}{\Gamma(\beta)} \int_{0}^{x}\left(x p-\zeta^{\prime}\right)^{\beta-1} \mathbb{E}_{\beta}\left(-\left(\frac{\zeta^{\prime}}{p}\right)^{\beta}\right) \bar{\phi}\left(\zeta^{\prime} ; \varepsilon\right) d \zeta .
$$

Recalling Definition 3.2, above equations reduce to

$$
\mathrm{S}[\phi(x ; \varepsilon)]=[S[\underline{\phi}(\mathrm{x} ; \varepsilon)], S[\bar{\phi}(\mathrm{x} ; \varepsilon)]]=\left[\frac{1}{\mathrm{p}^{\beta}} L[\underline{\phi}(\mathrm{x} ; \varepsilon)]=\frac{1}{\mathrm{p}}, \frac{1}{\mathrm{p}^{\beta}} L[\bar{\phi}(\mathrm{x} ; \varepsilon)]=\frac{1}{\mathrm{p}}\right]=\frac{1}{\mathrm{p}^{\beta}} \mathbb{L}[\phi(\mathrm{x})]=\frac{1}{\mathrm{p}} .
$$

Formula 3.4. For any constant c,

$$
S[\underline{\phi}(\mathrm{c} x ; \varepsilon)]=S[\underline{\phi}(\mathrm{x} ; \varepsilon)]_{=\mathrm{cp}}, S[\bar{\phi}(\mathrm{c} x ; \varepsilon)]=S[\bar{\phi}(\mathrm{x} ; \varepsilon)]_{=\mathrm{cp}} .
$$

Proof. By means of Definition 3.1, taking FST of $\underline{\phi}(\mathrm{cx} ; \varepsilon)$ and $\bar{\phi}(\mathrm{cx} ; \varepsilon)$, we get

$$
S[\underline{\phi}(\mathrm{c} x ; \varepsilon)]=\frac{1}{\mathrm{p}^{\beta}} \lim _{x \rightarrow \infty} \frac{1}{\Gamma(\beta)} \int_{0}^{x}(x-\zeta)^{\beta-1} \mathbb{E}_{\beta}\left(-\zeta^{\beta}\right) \underline{\phi}(\mathrm{pc} \zeta ; \varepsilon) \mathrm{d} \zeta
$$

and

$$
S[\bar{\phi}(\mathrm{cx} ; \varepsilon)]=\frac{1}{\mathrm{p}^{\beta}} \lim _{x \rightarrow \infty} \frac{1}{\Gamma(\beta)} \int_{0}^{x}(x-\zeta)^{\beta-1} \mathbb{E}_{\beta}\left(-\zeta^{\beta}\right) \bar{\phi}(\mathrm{pc} \zeta ; \varepsilon) \mathrm{d} \zeta,
$$

which implies

$$
\mathrm{S}[\underline{\phi}(\mathrm{c} x ; \varepsilon), \bar{\phi}(\mathrm{c} x ; \varepsilon)]=\mathrm{S}[\underline{\phi}(x ; \varepsilon), \bar{\phi}(x ; \varepsilon)]_{=c p} .
$$

\section{Formula 3.5.}

$$
\begin{aligned}
& S\left[\mathbb{E}_{\beta}\left(-c^{\beta} x^{\beta}\right) \underline{\phi}(x ; \varepsilon)\right]=\frac{1}{(1+c p)^{\beta}} S[\underline{\phi}(x ; \varepsilon)]=\frac{p}{1+c p}, \\
& S\left[\mathbb{E}_{\beta}\left(-c^{\beta} x^{\beta}\right) \bar{\phi}(x ; \varepsilon)\right]=\frac{1}{(1+c p)^{\beta}} S[\bar{\phi}(x ; \varepsilon)]=\frac{p}{1+c p} .
\end{aligned}
$$

Proof. Applying FST on $\mathbb{E}_{\beta}\left(-c^{\beta} x^{\beta}\right) \underline{\phi}(x ; \varepsilon)$ and $\mathbb{E}_{\beta}\left(-c^{\beta} x^{\beta}\right) \bar{\phi}(x ; \varepsilon)$, we attain

$$
\begin{aligned}
& S\left[\mathbb{E}_{\beta}\left(-c^{\beta} x^{\beta}\right) \underline{\phi}(x ; \varepsilon)\right]=\lim _{x \rightarrow \infty} \frac{1}{\Gamma(\beta)} \int_{0}^{x}(x-\zeta)^{\beta-1} \mathbb{E}_{\beta}\left(-\zeta^{\beta}\right) \mathbb{E}_{\beta}\left(-c^{\beta}(p \zeta)^{\beta}\right) \underline{\phi}(p \zeta ; \varepsilon) d \zeta, \\
& S\left[\mathbb{E}_{\beta}\left(-c^{\beta} x^{\beta}\right) \bar{\phi}(x ; \varepsilon)\right]=\lim _{x \rightarrow \infty} \frac{1}{\Gamma(\beta)} \int_{0}^{x}(x-\zeta)^{\beta-1} \mathbb{E}_{\beta}\left(-\zeta^{\beta}\right) \mathbb{E}_{\beta}\left(-c^{\beta}(p \zeta)^{\beta}\right) \bar{\phi}(p \zeta ; \varepsilon) d \zeta .
\end{aligned}
$$

Let $(1+\mathrm{cp}) \zeta=\zeta^{\prime}$ and $(1+\mathrm{cp}) \mathrm{d} \zeta=\mathrm{d} \zeta^{\prime}$, re-assuming Definition 3.1, we reach to the following equations,

$$
S\left[\mathbb{E}_{\beta}\left(-c^{\beta} x^{\beta}\right) \underline{\phi}(x ; \varepsilon)\right]=\frac{1}{(1+c p)^{\beta}} S[\underline{\phi}(x ; \varepsilon)]=\frac{p}{1+c p}
$$


and

$$
S\left[\mathbb{E}_{\beta}\left(-c^{\beta} \chi^{\beta}\right) \bar{\phi}(x ; \varepsilon)\right]=\frac{1}{(1+c p)^{\beta}} S[\bar{\phi}(x ; \varepsilon)]=\frac{p}{1+c p} .
$$

Theorem 3.6. Let $\mathrm{S}[\phi(x)]$ be the FFST of $\phi \in C_{\mathrm{f}}[\mathrm{a}, \mathrm{b}] \cap \boldsymbol{L}_{\mathrm{f}}[\mathrm{a}, \mathrm{b}]$, then the FFST of the Riemann-Liouville $H$-derivative of order $n-1 \leqslant \beta<n$ of fuzzy-valued function $\phi(x)$ is articulated as

$$
\mathrm{S}\left[\mathrm{RL} \mathfrak{D}_{\mathrm{a}^{+}}^{\beta} \phi(x)\right]=\frac{\mathrm{S}[\phi(x)]}{p^{\beta}} \Theta \frac{\mathrm{RL} \mathfrak{D}_{a^{+}}^{\beta-1} \phi(0)}{p},
$$

if $\phi(x)$ is I-differentiable and

$$
\mathrm{S}\left[R L_{\left.\mathfrak{D}_{a^{+}}^{\beta} \phi(x)\right]=-\frac{R L}{\mathfrak{D}_{a^{+}}^{\beta-1} \phi(0)}}^{p} \Theta\left(-\frac{S[\phi(x)]}{p^{\beta}}\right)\right.
$$

if $\phi(x)$ is II-differentiable.

Proof. Let $\phi(x)$ be I-differentiable for $\varepsilon \in[0,1]$, then we have

$$
\frac{S[\phi(x)]}{p^{\beta}} \Theta \frac{{ }^{R L} \mathfrak{D}_{a^{+}}^{\beta-1} \phi(0)}{p}=\left[\frac{S[\underline{\phi}(x ; \varepsilon)]}{p^{\beta}}-\frac{{ }^{R L} D_{a^{+}}^{\beta-1} \underline{\phi}(0 ; \varepsilon)}{p}, \frac{S[\bar{\phi}(x ; \varepsilon)]}{p^{\beta}}-\frac{{ }^{R L} D_{a^{+}}^{\beta-1} \bar{\phi}(0 ; \varepsilon)}{p}\right],
$$

since

$$
{ }^{R L} \mathfrak{D}_{a^{+}}^{\beta} \phi(x)=\left[{ }^{R L} D_{a^{+}}^{\beta} \phi(x ; \varepsilon), \overline{R L} D_{a^{+}}^{\beta} \phi(x ; \varepsilon)\right]=\left[{ }^{R L} D_{a^{+}}^{\beta} \underline{\phi}(x ; \varepsilon),{ }^{R L} D_{a^{+}}^{\beta} \bar{\phi}(x ; \varepsilon)\right] .
$$

Taking FST of lower and upper functions in (3.4) we get

$$
S\left[\underline{\mathrm{RL} D_{\mathbf{a}^{+}}^{\beta} \phi}(x ; \varepsilon)\right]=S\left[\mathrm{RL}^{\beta} \mathrm{a}^{+} \underline{\phi}(\mathrm{x} ; \varepsilon)\right]=\frac{S[\underline{\phi}(\mathrm{x} ; \varepsilon)]}{\mathrm{p}^{\beta}}-\frac{{ }^{\mathrm{RL}} D_{\mathbf{a}^{+}}^{\beta-1} \underline{\phi}(0 ; \varepsilon)}{p}
$$

and

$$
S\left[\overline{{ }^{R L} D_{a^{+}}^{\beta} \phi}(x ; \varepsilon)\right]=S\left[{ }^{R L} D_{a^{+}}^{\beta} \bar{\phi}(x ; \varepsilon)\right]=\frac{S[\bar{\phi}(x ; \varepsilon)]}{p^{\beta}}-\frac{{ }^{R L} D_{\mathbf{a}^{+}}^{\beta-1} \bar{\phi}(0 ; \varepsilon)}{p},
$$

respectively. Thus, substituting (3.5) and (3.6) in (3.3), it reduces to

$$
\frac{\mathrm{S}[\phi(x)]}{p^{\beta}} \Theta \frac{{ }^{R L} \mathfrak{D}_{a^{+}}^{\beta-1} \phi(0)}{p}=\left[S\left[{ }^{R L} D_{a^{+}}^{\beta} \underline{\phi}(x ; \varepsilon)\right], S\left[{ }^{R L} D_{a^{+}}^{\beta} \bar{\phi}(x ; \varepsilon)\right]\right]
$$

as $\mathrm{S}$ is linear, so

$$
\frac{\mathrm{S}[\phi(x)]}{p^{\beta}} \Theta \frac{\mathrm{RL}_{\mathfrak{D}_{a^{+}}^{\beta-1} \phi(0)}^{p}}{p}=\mathrm{S}\left[{ }^{\mathrm{RL}} D_{a^{+}}^{\beta} \underline{\phi}(x ; \varepsilon),{ }^{R L} D_{a^{+}}^{\beta} \bar{\phi}(x ; \varepsilon)\right] .
$$

Using the equality (3.4) on the right hand side, we get the required equation as



Now, assume that $\phi(x)$ is II-differentiable, then for $\varepsilon \in[0,1]$ we have

$$
-\frac{\mathrm{RL}_{\mathfrak{D}_{\mathrm{a}^{+}}^{\beta-1} \phi(0)}}{p} \Theta\left(-\frac{\mathrm{S}[\phi(x)]}{\mathrm{p}^{\beta}}\right)=\left[-\frac{\mathrm{RL}^{\beta} D_{\mathbf{a}^{+}}^{\beta-1} \bar{\phi}(0 ; \varepsilon)}{p}+\frac{S[\bar{\phi}(x ; \varepsilon)]}{p^{\beta}},-\frac{{ }^{\mathrm{RL}} D_{a^{+}}^{\beta-1} \underline{\phi}(0 ; \varepsilon)}{p}+\frac{S[\phi(x ; \varepsilon)]}{p^{\beta}}\right],
$$

since

$$
{ }^{R L} \mathfrak{D}_{a^{+}}^{\beta} \phi(x)=\left[\overline{R L} D_{a^{+}}^{\beta} \phi(x ; \varepsilon),{ }^{R L} D_{a^{+}}^{\beta} \phi(x ; \varepsilon)\right]=\left[{ }^{R L} D_{a^{+}}^{\beta} \bar{\phi}(x ; \varepsilon),{ }^{R L} D_{a^{+}}^{\beta} \underline{\phi}(x ; \varepsilon)\right] .
$$


Taking FST of lower and upper functions in (3.8) and on substituting the transformed functions in (3.7) we attain

$$
-\frac{{ }^{R L} \mathfrak{D}_{a^{+}}^{\beta-1} \phi(0)}{p} \Theta\left(-\frac{S[\phi(x)]}{p^{\beta}}\right)=\left[S\left[{ }^{R L} D_{a^{+}}^{\beta} \bar{\phi}(x ; \varepsilon)\right], S\left[{ }^{R L} D_{a^{+}}^{\beta} \underline{\phi}(x ; \varepsilon)\right]\right] .
$$

Consequently, we come up with the following expression

$$
-\frac{R L \mathfrak{D}_{a^{+}}^{\beta-1} \phi(0)}{p} \Theta\left(-\frac{S[\phi(x)]}{p^{\beta}}\right)=S\left[R L \mathfrak{D}_{a^{+}}^{\beta} \phi(x)\right] .
$$

\section{Fuzzy fractional differential equations}

Consider the following initial value problem of fractional order $0<\beta<1$ of fuzzy-valued function $\phi \in C_{\mathrm{f}}[\mathrm{a}, \mathrm{b}] \cap \boldsymbol{L}_{\mathrm{f}}[\mathrm{a}, \mathrm{b}]$,

$$
\mathrm{RL}_{\mathfrak{D}_{\mathbf{a}^{+}}^{\beta} \phi(x)=g(x, \phi(x)),}
$$

subjected to

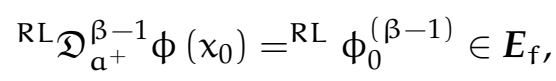

where $g(x, \phi(x))$ is linear fuzzy-valued function. In order to solve the above FFDE, together with all cases of Riemann-Liouville H-differentiability, we apply FFST on both sides of (4.1), that is

$$
\mathrm{S}\left[\mathrm{RL} \mathfrak{D}_{\mathrm{a}^{+}}^{\beta} \phi(x)\right]=\mathrm{S}[g(x, \phi(x))] .
$$

Consider $\phi(x)$ is an I-differentiable function and let (4.2) be expanded to its lower and upper functions, then, for $0 \leqslant \varepsilon \leqslant 1$

$$
\begin{aligned}
& S[g(x, \underline{\phi}(x ; \varepsilon))]=\frac{S[\underline{\phi}(x ; \varepsilon)]}{p^{\beta}}-\frac{{ }^{R L} D_{a^{+}}^{\beta-1} \underline{\phi}(0 ; \varepsilon)}{p}, \\
& S[g(x, \bar{\phi}(x ; \varepsilon))]=\frac{S[\phi(x ; \varepsilon)]}{p^{\beta}}-\frac{{ }^{R L} D_{a^{+}}^{\beta-1} \bar{\phi}(0 ; \varepsilon)}{p},
\end{aligned}
$$

where

$$
g(x, \underline{\phi}(x ; \varepsilon))=\min \{(x, v) \mid v \in[\underline{\phi}(x ; \varepsilon), \bar{\phi}(x ; \varepsilon)]\}
$$

and

$$
g(x, \bar{\phi}(x ; \varepsilon))=\max \{(x, v) \mid v \in[\underline{\phi}(x ; \varepsilon), \bar{\phi}(x ; \varepsilon)]\}
$$

Let

$$
S[\underline{\phi}(x ; \varepsilon)]=Q_{1}(p ; \varepsilon), \quad S[\bar{\phi}(x ; \varepsilon)]=T_{1}(p ; \varepsilon),
$$

where $Q_{1}(p ; \varepsilon)$ and $T_{1}(p ; \varepsilon)$ are solutions of (4.4) and (4.5), respectively. Then, on using inverse FST, $\underline{\phi}(x ; \varepsilon)$ and $\bar{\phi}(x ; \varepsilon)$ are figured out as

$$
\underline{\phi}(x ; \varepsilon)=S^{-1}\left[Q_{1}(p ; \varepsilon)\right], \quad \bar{\phi}(x ; \varepsilon)=S^{-1}\left[T_{1}(p ; \varepsilon)\right] .
$$

Now, let $\phi(x)$ be a II-differential function, then, for $0 \leqslant \varepsilon \leqslant 1$, (4.3) expands to

$$
\begin{aligned}
& S[g(x, \underline{\phi}(x ; \varepsilon))]=\frac{S[\bar{\phi}(x ; \varepsilon)]}{p^{\beta}}-\frac{\mathrm{RL}^{\beta} D_{\mathrm{a}^{+}}^{\beta-1} \bar{\phi}(0 ; \varepsilon)}{p}, \\
& S[g(x, \bar{\phi}(x ; \varepsilon))]=\frac{S[\underline{\phi}(x ; \varepsilon)]}{p^{\beta}}-\frac{{ }^{\beta L} D_{a^{+}}^{\beta-1} \underline{\phi}(0 ; \varepsilon)}{p},
\end{aligned}
$$


where $g(x, \underline{\phi}(x ; \varepsilon))$ and $g(x, \bar{\phi}(x ; \varepsilon))$ are same as in (4.6) and (4.7). Let

$$
S[\underline{\phi}(x ; \varepsilon)]=Q_{2}(p ; \varepsilon), \quad S[\bar{\phi}(x ; \varepsilon)]=T_{2}(p ; \varepsilon),
$$

where, $Q_{1}(p ; \varepsilon)$ and $T_{1}(p ; \varepsilon)$ are solutions of (4.8) and (4.9), respectively. Then, on using inverse FST, $\underline{\phi}(x ; \varepsilon)$ and $\bar{\phi}(\chi ; \varepsilon)$ are figured out as

$$
\underline{\Phi}(x ; \varepsilon)=S^{-1}\left[Q_{2}(p ; \varepsilon)\right], \quad \bar{\phi}(x ; \varepsilon)=S^{-1}\left[T_{2}(p ; \varepsilon)\right] .
$$

\section{Illustrative examples}

In this section, explanatory examples of FFDEs considered under Riemann-Liouville H-differentiability are presented and analyzed using the proposed transform.

Example 5.1. Consider the following FFDE

$$
\mathrm{RL}_{\mathfrak{D}_{0^{+}}^{\beta}} \mathrm{y}(x)=\lambda y(x),
$$

subjected to

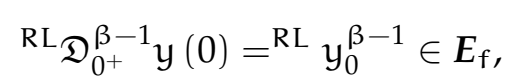

where, $y(x) \in C_{f}[a, b] \cap L_{f}[a, b]$ and $\lambda \in \mathfrak{R}$. Now, for the case (i), let $y(x)$ be I-differentiable and $\lambda \in \mathfrak{R}^{+}$, then applying FFST on both sides of (5.1) we obtain

$$
S\left[R L \mathfrak{D}_{0^{+}}^{\beta} y(x)\right]=\lambda S[y(x)] .
$$

Using Theorem 3.6, we get

$$
\frac{S[\underline{y}(x ; \varepsilon)]}{p^{\beta}}-\frac{{ }^{R L} D_{0^{+}}^{\beta-1} \underline{y}(0 ; \varepsilon)}{p}=\lambda S[\underline{y}(x ; \varepsilon)], \quad \frac{S[\bar{y}(x ; \varepsilon)]}{p^{\beta}}-\frac{{ }^{R L} D_{0^{+}}^{\beta-1} \bar{y}(0 ; \varepsilon)}{p}=\lambda S[\bar{y}(x ; \varepsilon)],
$$

which implies

$$
S[\underline{y}(x ; \varepsilon)]\left[\frac{1}{p^{\beta}}-\lambda\right]=\frac{{ }^{R L} D_{0^{+}}^{\beta-1} \underline{y}(0 ; \varepsilon)}{p}, \quad S[\bar{y}(x ; \varepsilon)]\left[\frac{1}{p^{\beta}}-\lambda\right]=\frac{{ }^{R L} D_{0^{+}}^{\beta-1} \bar{y}(0 ; \varepsilon)}{p},
$$

simplifying and on applying inverse FST, we attain

$$
\underline{y}(x ; \varepsilon)={ }^{R L} D_{0^{+}}^{\beta-1} \underline{y}(0 ; \varepsilon) S^{-1}\left[\frac{p^{\beta-1}}{1-\lambda p^{\beta}}\right], \quad \bar{y}(x ; \varepsilon)={ }^{R L} D_{0^{+}}^{\beta-1} \bar{y}(0 ; \varepsilon) S^{-1}\left[\frac{p^{\beta-1}}{1-\lambda p^{\beta}}\right],
$$

using (5.2) and the subsequent equality [7],

$$
S^{-1}\left[\frac{u^{\gamma-1}}{1-\omega u^{\beta}}\right]=x^{\gamma-1} \mathbb{E}_{\beta, \gamma}^{\delta}\left(\omega x^{\beta}\right),
$$

we come up with the following solutions of the aforementioned FFDE,

$$
\underline{y}(x ; \varepsilon)={ }^{R L} \underline{y}_{0}^{\beta-1}\left(x^{\beta-1} \mathbb{E}_{\beta, \beta}\left(\lambda x^{\beta}\right)\right), \bar{y}(x ; \varepsilon)={ }^{R L} \bar{y}_{0}^{\beta-1}\left(x^{\beta-1} \mathbb{E}_{\beta, \beta}\left(\lambda x^{\beta}\right)\right) .
$$

In addition, if $\lambda \in \mathfrak{R}^{-}$, then (5.3) expands to its lower and upper functions as

$$
\frac{S[\underline{y}(x ; \varepsilon)]}{p^{\beta}}-\frac{{ }^{R L} D_{0^{+}}^{\beta-1} \underline{y}(0 ; \varepsilon)}{p}=\lambda S[\bar{y}(x ; \varepsilon)], \quad \frac{S[\bar{y}(x ; \varepsilon)]}{p^{\beta}}-\frac{{ }^{R L} D_{0^{+}}^{\beta-1} \bar{y}(0 ; \varepsilon)}{p}=\lambda S[\underline{y}(x ; \varepsilon)] .
$$


Hence, following the above simplifications, the solutions for the negative $\lambda$ can be expressed as

$$
\begin{aligned}
& \underline{y}(x ; \varepsilon)={ }^{R L} \underline{y}_{0}^{\beta-1}\left(x^{\beta-1} \mathbb{E}_{2 \beta, \beta}\left(\lambda^{2} \chi^{2 \beta}\right)\right)+\lambda^{R L} \bar{y}_{0}^{\beta-1}\left(x^{2 \beta-1} \mathbb{E}_{2 \beta, 2 \beta}\left(\lambda^{2} x^{2 \beta}\right)\right), \\
& \bar{y}(x ; \varepsilon)={ }^{R L} \bar{y}_{0}^{\beta-1}\left(x^{\beta-1} \mathbb{E}_{2 \beta, \beta}\left(\lambda^{2} x^{2 \beta}\right)\right)+\lambda^{R L} \underline{y}_{0}^{\beta-1}\left(x^{2 \beta-1} \mathbb{E}_{2 \beta, 2 \beta}\left(\lambda^{2} x^{2 \beta}\right)\right) .
\end{aligned}
$$

In case of II-differentiability of $y(x)$, the solutions determined are similar to (5.5) and (5.6) for $\lambda \in \mathfrak{R}^{-}$ and $\lambda \in \mathfrak{R}^{+}$, respectively. Besides, let $\mathrm{RL}_{\mathfrak{D}_{0^{+}}^{\beta-1}} \mathrm{y}(0)=[1+\varepsilon, 3-\varepsilon]$ and $\lambda=1$, then (5.5) becomes

$$
\underline{y}(x ; \varepsilon)=(1+\varepsilon)\left(x^{\beta-1} \mathbb{E}_{\beta, \beta}\left(x^{\beta}\right)\right), \bar{y}(x ; \varepsilon)=(3-\varepsilon)\left(x^{\beta-1} \mathbb{E}_{\beta, \beta}\left(x^{\beta}\right)\right),
$$

whereas, for $\lambda=-1$, (5.6) becomes

$$
\begin{aligned}
& \underline{y}(x ; \varepsilon)=(1+\varepsilon)\left(x^{\beta-1} \mathbb{E}_{2 \beta, \beta}\left(x^{2 \beta}\right)\right)-(3-\varepsilon)\left(x^{2 \beta-1} \mathbb{E}_{2 \beta, 2 \beta}\left(x^{2 \beta}\right)\right), \\
& \bar{y}(x ; \varepsilon)=(3-\varepsilon)\left(x^{\beta-1} \mathbb{E}_{2 \beta, \beta}\left(x^{2 \beta}\right)\right)-(1+\varepsilon)\left(x^{2 \beta-1} \mathbb{E}_{2 \beta, 2 \beta}\left(x^{2 \beta}\right)\right) .
\end{aligned}
$$

Example 5.2. Consider the following FFDE,

$$
\mathrm{RL}_{\mathfrak{D}_{0^{+}}^{\beta}} \mathrm{y}(x)=-y(x)+\frac{2}{\Gamma(3-\beta)} x^{2-\beta}-\frac{1}{\Gamma(2-\beta)} x^{1-\beta}+x^{2}-x,
$$

subjected to

$$
\mathrm{RL}_{\mathfrak{D}^{+}}^{\beta-1} \mathrm{y}(0)={ }^{\mathrm{RL}} \mathrm{y}_{0}^{\beta-1} \in \boldsymbol{E}_{\mathrm{f}} .
$$

Suppose $y(x)$ is I-differentiable fuzzy-valued function, then applying FFST on both sides of (5.7), we obtain

$$
S\left[R L \mathfrak{D}_{0^{+}}^{\beta} y(x)\right]=S\left[-y(x)+\frac{2}{\Gamma(3-\beta)} x^{2-\beta}-\frac{1}{\Gamma(2-\beta)} x^{1-\beta}+x^{2}-x\right]
$$

and in terms of lower and upper functions, for $0 \leqslant \varepsilon \leqslant 1$,

$$
\begin{aligned}
& S\left[{ }^{\mathrm{RL}} D_{0^{+}}^{\beta} \underline{y}(x ; \varepsilon)\right]=-S[\bar{y}(x ; \varepsilon)]+\frac{2}{\Gamma(3-\beta)} S\left[x^{2-\beta}\right]-\frac{1}{\Gamma(2-\beta)} S\left[x^{1-\beta}\right]+S\left[x^{2}\right]-S[x], \\
& S\left[{ }^{\mathrm{RL}} D_{0^{+}}^{\beta} \overline{\mathrm{y}}(\mathrm{x} ; \varepsilon)\right]=-S[\underline{y}(\mathrm{x} ; \varepsilon)]+\frac{2}{\Gamma(3-\beta)} S\left[x^{2-\beta}\right]-\frac{1}{\Gamma(2-\beta)} S\left[x^{1-\beta}\right]+S\left[x^{2}\right]-S[x] .
\end{aligned}
$$

Using Theorem 3.6, we get

$$
\begin{aligned}
& \frac{S[\underline{y}(x ; \varepsilon)]}{p^{\beta}}-\frac{{ }^{R L} D_{0^{+}}^{\beta-1} \underline{y}(0 ; \varepsilon)}{p}=-S[\bar{y}(x ; \varepsilon)]+2 p^{2-\beta}-p^{1-\beta}+2 p^{2}-p, \\
& \frac{S[\bar{y}(x ; \varepsilon)]}{p^{\beta}}-\frac{{ }^{R L} D_{0^{+}}^{\beta-1} \bar{y}(0 ; \varepsilon)}{p}=-S[\underline{y}(x ; \varepsilon)]+2 p^{2-\beta}-p^{1-\beta}+2 p^{2}-p .
\end{aligned}
$$

On undergoing some manipulations and using the initial condition specified in (5.8), we acquire

$$
\begin{aligned}
& S[\underline{y}(x ; \varepsilon)]={ }^{R L} \underline{y}_{0}^{\beta-1}\left[\frac{p^{\beta-1}}{1-p^{2 \beta}}\right]-{ }^{R L} \bar{y}_{0}^{\beta-1}\left[\frac{p^{2 \beta-1}}{1-p^{2 \beta}}\right]+2 p^{2}-p, \\
& S[\bar{y}(x ; \varepsilon)]={ }^{R L} \bar{y}_{0}^{\beta-1}\left[\frac{p^{\beta-1}}{1-p^{2 \beta}}\right]-{ }^{R L} \underline{y}_{0}^{\beta-1}\left[\frac{p^{2 \beta-1}}{1-p^{2 \beta}}\right]+2 p^{2}-p .
\end{aligned}
$$

Finally, using (5.4) and inverse FST, we get the solutions as

$$
\begin{aligned}
& \underline{y}(x ; \varepsilon)={ }^{R L} \underline{y}_{0}^{\beta-1}\left(x^{\beta-1} \mathbb{E}_{2 \beta, \beta}\left(x^{2 \beta}\right)\right)-{ }^{R L} \bar{y}_{0}^{\beta-1}\left(x^{2 \beta-1} \mathbb{E}_{2 \beta, 2 \beta}\left(x^{2 \beta}\right)\right)+x^{2}-x, \\
& \bar{y}(x ; \varepsilon)={ }^{R L} \bar{y}_{0}^{\beta-1}\left(x^{\beta-1} \mathbb{E}_{2 \beta, \beta}\left(x^{2 \beta}\right)\right)-{ }^{R L} \underline{y}_{0}^{\beta-1}\left(x^{2 \beta-1} \mathbb{E}_{2 \beta, 2 \beta}\left(x^{2 \beta}\right)\right)+x^{2}-x .
\end{aligned}
$$


Whereas, in case of II-differentiability of $y(x),(5.9)$ changes to,

$$
\begin{aligned}
& \frac{S[\bar{y}(x ; \varepsilon)]}{p^{\beta}}-\frac{{ }^{R L} D_{0^{+}}^{\beta-1} \bar{y}(0 ; \varepsilon)}{p}=-S[\bar{y}(x ; \varepsilon)]+2 p^{2-\beta}-p^{1-\beta}+2 p^{2}-p, \\
& \frac{S[\underline{y}(x ; \varepsilon)]}{p^{\beta}}-\frac{{ }^{R L} D_{0^{+}}^{\beta-1} \underline{y}(0 ; \varepsilon)}{p}=-S[\underline{y}(x ; \varepsilon)]+2 p^{2-\beta}-p^{1-\beta}+2 p^{2}-p .
\end{aligned}
$$

Hence, following the above simplifications, the solutions in this case can be constructed as

$$
\begin{aligned}
& \underline{y}(x ; \varepsilon)={ }^{R L} \underline{y}_{0}^{\beta-1}\left(x^{\beta-1} \mathbb{E}_{\beta, \beta}\left(-x^{\beta}\right)\right)+x^{2}-x, \\
& \bar{y}(x ; \varepsilon)={ }^{R L} \bar{y}_{0}^{\beta-1}\left(x^{\beta-1} \mathbb{E}_{\beta, \beta}\left(-x^{\beta}\right)\right)+x^{2}-x .
\end{aligned}
$$

Moreover, let ${ }^{\mathrm{RL}} D_{0^{+}}^{\beta-1} \mathrm{y}(0)=[1+\varepsilon, 3-\varepsilon]$, then (5.10) becomes

$$
\begin{aligned}
& \underline{y}(x ; \varepsilon)=(1+\varepsilon)\left(x^{\beta-1} \mathbb{E}_{2 \beta, \beta}\left(x^{2 \beta}\right)\right)-(3-\varepsilon)\left(x^{2 \beta-1} \mathbb{E}_{2 \beta, 2 \beta}\left(x^{2 \beta}\right)\right)+x^{2}-x, \\
& \bar{y}(x ; \varepsilon)=(3-\varepsilon)\left(x^{\beta-1} \mathbb{E}_{2 \beta, \beta}\left(x^{2 \beta}\right)\right)-(1+\varepsilon)\left(x^{2 \beta-1} \mathbb{E}_{2 \beta, 2 \beta}\left(x^{2 \beta}\right)\right)+x^{2}-x,
\end{aligned}
$$

while, (5.11) becomes

$$
\underline{y}(x ; \varepsilon)=(1+\varepsilon)\left(\chi^{\beta-1} \mathbb{E}_{\beta, \beta}\left(-\chi^{\beta}\right)\right)+x^{2}-x, \quad \bar{y}(x ; \varepsilon)=(3-\varepsilon)\left(\chi^{\beta-1} \mathbb{E}_{\beta, \beta}\left(-\chi^{\beta}\right)\right)+x^{2}-\chi .
$$

\section{Conclusion}

In this description, discovering comprehensive importance of analytical solutions of FFDEs in physics and engineer fields, we proposed FFST as a combination of FST and fuzzy theory. Illustratively, the solutions of some homogeneous and non-homogeneous FFDEs were determined. Thus, the whole study can be wrapped up with the following results.

- After constructing the properties of FFST for fuzzy-valued functions, its unit preserving property remained unchanged that verifies its appropriateness for the transformation of fuzzy-valued functions.

- The Riemann-Liouville Hukuhara-derivatives of fuzzy-valued functions has a pragmatic importance in fuzzy calculus and constructive in giving better solutions of the functions that reflects the realworld problems. Therefore, elucidations of FFST of these derivatives added a useful contribution to this theory.

- The analytical solutions of FFDEs, obtained by FFST, for different cases of Riemann-Liouville Hdifferentiability of fuzzy-valued functions were found to be exactly same as the solutions in [20], which further shows the effectiveness of FFST for FFDEs.

As a result, FFST is concluded to be an efficient, appropriate, and easy computational tool for analytical solutions of FFDEs. For future studies, we are seeking to reveal many other interesting properties and applications of fuzzy fractional Sumudu transform.

\section{Acknowledgment}

The authors are thankful to the Dean faculty of Science, University of Karachi for supporting this work and the referees for their suggestions for the improvement of this paper. 


\section{References}

[1] R. P. Agarwal, V. Lakshmikantham, J. J. Nieto, On the concept of solution for fractional differential equations with uncertainty, Nonlinear Anal., 72 (2010), 2859-2862. 1

[2] G. A. Anastassiou, I. K. Argyros, S. Kumar, Monotone convergence of extended iterative methods and fractional calculus with applications, Fund. Inform., 151 (2017), 241-253. 1

[3] B. Bede, S. G. Gal, Generalizations of the differentiability of fuzzy-number-valued functions with applications to fuzzy differential equations, Fuzzy Sets and Systems, 151 (2005), 581-599. 1, 2.1, 2.2

[4] F. B. M. Belgacem, A. Karaballi, Sumudu transform fundamental properties investigations and applications, J. Appl. Math. Stoch. Anal., 2006 (2006), 23 pages. 1, 3

[5] F. B. M. Belgacem, R. Silambarasan, A distinctive Sumudu treatment of trigonometric functions, J. Comput. Appl. Math., 312 (2017), 74-81. 1

[6] H. Bulut, H. M. Baskonus, F. B. M. Belgacem, The analytical solution of some fractional ordinary differential equations by the Sumudu transform method, Abstr. Appl. Anal., 2013 (2013), 6 pages. 1

[7] R. Darzi, Sumudu transform method for solving fractional differential equations and fractional diffusion-wave equation, J. Math. Comput. Sci., 6 (2013), 79-84. 1, 5.1

[8] E. F. Doungmo Goufo, S. Kumar, Shallow water wave models with and without singular kernel: existence, uniqueness, and similarities, Math. Probl. Eng., 2017 (2017), 9 pages. 1

[9] F. Gao, H. M. Srivastava, Y.-N. Gao, Y.-X. Jun, A coupling method involving the Sumudu transform and the variational iteration method for a class of local fractional diffusion equations, J. Nonlinear Sci. Appl., 9 (2016), 5830-5835. 1

[10] V. G. Gupta, B. Sharma, A. Kilicman, A note on fractional Sumudu transform, J. Appl. Math., 2010 (2010), 9 pages. 1

[11] O. Kaleva, Fuzzy differential equations, Fuzzy Sets and Systems, 24 (1987), 301-317. 1, 2.1

[12] N. A. Khan, O. A. Razzaq, M. Ayaz, On the solution of fuzzy differential equations by fuzzy Sumudu transform, Nonlinear Eng., 4 (2015), 49-60. 1, 3

[13] N. A. Khan, F. Riaz, O. A. Razzaq, Numerical simulations for solving fuzzy fractional differential equations by max-min improved Euler method, J. Appl. Comput. Sci. Method., 7 (2015), 53-83. 1

[14] E. Khodadadi, M. Karabacak, Solving fuzzy fractional partial differential equations by fuzzy Laplace-Fourier transforms, J. Comput. Anal. Appl., 19 (2015), 260-271. 1

[15] A. Kiliçman, H. Eltayeb, K. A. M. Atan, A note on the comparison between Laplace and Sumudu transforms, Bull. Iranian Math. Soc., 37 (2011), 131-141. 1

[16] X. Liang, F. Gao, Y.-N. Gao, Y.-X. Jun, Applications of a novel integral transform to partial differential equations, J. Nonlinear Sci. Appl., 10 (2017), 528-534. 1

[17] K. S. Miller, B. Ross, An introduction to the fractional calculus and fractional differential equations, John Wiley \& Sons, New York, (1993). 1

[18] V. H. Ngo, Fuzzy fractional functional integral and differential equations, Fuzzy Sets and Systems, 280 (2015), 58-90. $1,2.2$

[19] T. Patel, R. Meher, A study on temperature distribution, efficiency and effectiveness of longitudinal porous fins by using Adomian decomposition Sumudu transform method, Proced. Eng., 127 (2015), 751-758. 1

[20] S. Salahshour, T. Allahviranaloo, S. Abbasbandy, Solving fuzzy fractional differential equations by fuzzy Laplace transforms, Commun. Nonlinear Sci. Numer. Simul., 17 (2012), 1372-1381. 1, 3, 6

[21] S. Tuluce Demiray, H. Bulut, F. B. M. Belgacem, Sumudu transform method for analytical solutions of fractional type ordinary differential equations, Math. Probl. Eng., 2015 (2015), 6 pages. 1

[22] G. K. Watugala, Sumudu transform: A new integral transform to solve differential equations and control engineering problems, Internat. J. Math. Ed. Sci. Tech., 24 (1993), 35-43. 1, 3

[23] L. A. Zadeh, Fuzzy sets, Information and Control, 8 (1965), 338-353. 1, 2.1

[24] L. A. Zadeh, Fuzzy sets as a basis for a theory of possibility, Fuzzy Sets and Systems, 1 (1978), 3-28. 1, 2.1 